LAWRENCE LIVERMORE N A TION A L LABORATORY

Resonant Auger Effect at High X-Ray Intensity

N. Rohringer, R. Santra

April 24, 2008

Physical Review A 
This document was prepared as an account of work sponsored by an agency of the United States government. Neither the United States government nor Lawrence Livermore National Security, LLC, nor any of their employees makes any warranty, expressed or implied, or assumes any legal liability or responsibility for the accuracy, completeness, or usefulness of any information, apparatus, product, or process disclosed, or represents that its use would not infringe privately owned rights. Reference herein to any specific commercial product, process, or service by trade name, trademark, manufacturer, or otherwise does not necessarily constitute or imply its endorsement, recommendation, or favoring by the United States government or Lawrence Livermore National Security, LLC. The views and opinions of authors expressed herein do not necessarily state or reflect those of the United States government or Lawrence Livermore National Security, LLC, and shall not be used for advertising or product endorsement purposes. 


\title{
Resonant Auger effect at high x-ray intensity
}

\author{
Nina Rohringer* and Robin Santra \\ Argonne National Laboratory, Argonne, Illinois 60439, USA
}

(Dated: March 31, 2008)

\begin{abstract}
The resonant Auger effect of atomic neon exposed to high-intensity x-ray radiation in resonance with the $1 s \rightarrow 3 p$ transition is discussed. High intensity here means that the x-ray peak intensity is sufficient $\left(\sim 10^{18} \mathrm{~W} / \mathrm{cm}^{2}\right)$ to induce Rabi oscillations between the neon ground state and the $1 s^{-1} 3 p\left({ }^{1} P\right)$ state within the relaxation lifetime of the inner-shell vacancy. For the numerical analysis presented, an effective two-level model, including a description of the resonant Auger decay process, is employed. Both coherent and chaotic x-ray pulses are treated. The latter are used to simulate radiation from x-ray free-electron lasers based on the principle of self-amplified spontaneous emission. Observing x-ray-driven atomic population dynamics in the time domain is challenging for chaotic pulse ensembles. A more practical option for experiments using x-ray free-electron lasers is to measure the line profiles in the kinetic energy distribution of the resonant Auger electron. This provides information on both atomic population dynamics and x-ray pulse properties.
\end{abstract}

PACS numbers: 32.80.Hd, 33.20.Xx, 41.60.Cr, 82.50.Kx

${ }^{*}$ Present address: Lawrence Livermore National Laboratory, Livermore, California 94551, USA 


\section{INTRODUCTION}

Since its discovery in 1980 [1], the resonant Auger effect [2, 3] and its influence on various experimental observables have been the subject of intense investigation [4-17]. In a simple picture, the resonant Auger effect may be interpreted as a resonant photoionization process mediated by a particle-hole configuration involving an inner-shell hole and an excited electron in a discrete one-electron state. The electronic decay of the inner-shell hole gives rise to an electron spectrum that is similar to an Auger electron spectrum. The excited electron generally does not participate in the Auger decay step. However, in contrast to regular Auger decay, where the photoexcited electron is completely removed from the parent ion, the presence of this electron in the resonant case leads to a characteristic modification of the positions of the Auger electron spectral lines. Further, for narrow-bandwidth x rays, the kinetic energy of a photoelectron associated with a given resonant Auger decay channel displays a linear dependence on the photon energy, and the width of the electron line profile is determined by the photon bandwidth, not by the Auger decay width.

Like all other x-ray-induced processes currently experimentally accessible, the resonant Auger effect studied so far is a first-order process with respect to the interaction with the photon field. In this paper we show how the resonant Auger effect will be modified at high $\mathrm{x}$-ray intensity. This topic is timely for two reasons. First, the x-ray free-electron lasers $[18,19]$ that are currently under construction [20-22] are expected to provide the intensity necessary to drive nonperturbative x-ray processes on a time scale that is comparable with the lifetime of an inner-shell hole [23]. Second, these fourth-generation synchrotron radiation sources are based on the principle of self-amplified spontaneous emission (SASE) [24]. As a consequence, the pulse properties of the x-ray radiation generated vary from shot to shot $[25,26]$. This limits the longitudinal coherence time near a photon energy of $1 \mathrm{keV}$ to a few femtoseconds [27], which is again comparable with Auger lifetimes. As shown in Ref. [28], this has interesting consequences for double-core-hole formation via x-ray twophoton absorption at high, nonresonant photon energies. Here, we discuss consequences for the high-intensity resonant Auger effect. Because the Linac Coherent Light Source (LCLS) [20] is the first of the x-ray free-electron lasers that is expected to come online, we focus in the following on its parameter regime [29]. Section II briefly reviews resonant Auger decay at the $1 s \rightarrow 3 p$ absorption resonance of atomic neon. In Sec. III, we describe the theoretical 
model we use to investigate how the presence of high-intensity $\mathrm{x}$ rays is reflected in the resonant Auger effect. The results of our computations on neon are presented in Sec. IV. Section V contains a summary. Atomic units are used throughout unless otherwise stated.

\section{THE $1 s \rightarrow 3 p$ RESONANCE IN NEON}

The LCLS will initially operate in the soft-x-ray regime above $800 \mathrm{eV}$. Since the $1 s$ ionization threshold of Ne lies at $870.2 \mathrm{eV}$ [30], it is planned to perform some of the first experiments at LCLS using atomic neon [23]. The most prominent and well-isolated preedge resonance in $\mathrm{Ne}$ is the excitation from the atomic ground state $\left({ }^{1} S\right)$ to the $1 s^{-1} 3 p$ $\left({ }^{1} P\right)$ state at a photon energy of $867.1 \mathrm{eV}$ [31-35]. Good agreement with the experimentally observed resonance profile [33] is obtained $[36,37]$ by assuming that the decay width $\Gamma_{1 s^{-1} 3 p}$ of the $1 s^{-1} 3 p$ state equals the Auger width $\Gamma_{1 s^{-1}}$ of $\mathrm{Ne}^{+} 1 s^{-1}$, i.e., to a good approximation $\Gamma_{1 s^{-1} 3 p}=\Gamma_{1 s^{-1}}=0.27 \mathrm{eV}[30]$. (Ab initio calculations indicate that $\Gamma_{1 s^{-1} 3 p}$ could be smaller than $\Gamma_{1 s^{-1}}$ by $\sim 0.01 \mathrm{eV}$ [33].) The $1 s^{-1} 3 p$ resonance state of Ne decays with a branching ratio of $64 \%$ into electronically bound states of $\mathrm{Ne}^{+}$plus a resonant Auger electron [34]. This is the case we will concentrate on in the following. Particularly important final states in this category are the $2 p^{-2}\left({ }^{1} D\right) 3 p$ (spectator) and $2 p^{-2}\left({ }^{1} D\right) 4 p$ (shake-up) states of $\mathrm{Ne}^{+}[31]$. They give rise to distinct peaks in the resonant Auger electron spectrum. Because of the relatively large Auger decay width, the ${ }^{2} P,{ }^{2} D$, and ${ }^{2} F$ terms associated with $2 p^{-2}\left({ }^{1} D\right) 3 p$ can only be partially resolved; they are unresolved for $2 p^{-2}\left({ }^{1} D\right) 4 p$ [31].

\section{THEORETICAL MODEL}

Even without employing a monochromator, the spectral bandwidth of LCLS near the $1 s \rightarrow 3 p$ absorption resonance is expected to be only a few hundred meV [29]. By choosing the carrier frequency $\omega_{\mathrm{x}}$ of LCLS to coincide with the resonance frequency, it will be possible to selectively excite the transition from the Ne ground state to the $1 s^{-1} 3 p$ state. We therefore adopt an effective two-level model, including a description of the resonant Auger decay process, to investigate the impact of high-intensity x-ray interaction. State $|1\rangle$ in this model

is the Ne ground state. State $|2\rangle$ is the closed-channel component of the $1 s^{-1} 3 p$ resonance wave function. By diagonalizing the exact many-electron Hamiltonian in a suitably selected 
subset of the electronic configuration space, the Coulomb-interaction-mediated coupling to the open Auger decay channels $\left|i, \varepsilon_{i}\right\rangle$ can be eliminated. Here, $\left|i, \varepsilon_{i}\right\rangle$ stands for the $i$ th eigenstate of $\mathrm{Ne}^{+}$plus a resonant Auger electron of energy $\varepsilon_{i}$. Employing Wigner-Weisskopf theory $[38,39]$, the partial decay width $\Gamma_{i}$ associated with the irreversible transition from $|2\rangle$ to $\left|i, \varepsilon_{i}\right\rangle$ is obtained as

$$
\Gamma_{i}=2 \pi\left|\left\langle i, \varepsilon_{i}\left|\hat{V}_{\mathrm{C}}\right| 2\right\rangle\right|^{2},
$$

where $\hat{V}_{\mathrm{C}}$ symbolizes the Coulomb two-body operator, and $\left|i, \varepsilon_{i}\right\rangle$ is assumed to be energynormalized. Thus, the Coulomb coupling matrix element between $|2\rangle$ and $\left|i, \varepsilon_{i}\right\rangle$ is determined by $\Gamma_{i}$ up to a phase $\varphi_{i}$ :

$$
\left\langle i, \varepsilon_{i}\left|\hat{V}_{\mathrm{C}}\right| 2\right\rangle=\sqrt{\frac{\Gamma_{i}}{2 \pi}} e^{i \varphi_{i}} .
$$

We assume that the x-ray field is linearly polarized along the $z$ axis with field strength

$$
\mathcal{E}(t)=\mathcal{E}_{\mathrm{c}}(t) \cos \left(\omega_{\mathrm{x}} t\right)+\mathcal{E}_{\mathrm{s}}(t) \sin \left(\omega_{\mathrm{x}} t\right),
$$

where $\mathcal{E}_{\mathrm{c}}(t)$ and $\mathcal{E}_{\mathrm{s}}(t)$ are slowly varying on the time scale of $2 \pi / \omega_{\mathrm{x}}$. Thus, the cycle-averaged intensity is

$$
I(t)=\frac{1}{8 \pi \alpha}\left\{\mathcal{E}_{\mathrm{c}}^{2}(t)+\mathcal{E}_{\mathrm{s}}^{2}(t)\right\} .
$$

In this expression, $\alpha$ is the fine-structure constant, and the intensity is measured in units of $I_{0}=E_{\mathrm{h}} /\left(t_{0} a_{0}^{2}\right)=6.43641 \times 10^{15} \mathrm{~W} / \mathrm{cm}^{2}$ ( $E_{\mathrm{h}}$ is the Hartree energy, $t_{0}$ the atomic unit of time, and $a_{0}$ the Bohr radius).

In the calculations presented in Sec. IV, we consider two scenarios for $\mathcal{E}(t)$. In the first scenario, in order to simulate the statistical nature of SASE free-electron laser pulses (see Sec. I), we adopt an approach developed in Ref. [40]. Within this model, the Fourier expansion coefficients of the electric field in $\omega$ space are each Gaussian random variables with zero mean and a variance equal to the power spectrum at the corresponding frequency. We choose a Gaussian power spectrum centered at the frequency $\omega_{\mathrm{x}}$ with a variance corresponding to the relative LCLS gain bandwidth of $4.4 \times 10^{-4}$ [41]. For each simulated pulse, the Fourier expansion coefficients of the electric field in $\omega$ space are selected according to their respective probability distribution. The fields $\mathcal{E}_{\mathrm{c}}(t)$ and $\mathcal{E}_{\mathrm{s}}(t)$ are then determined by applying the fast Fourier transform algorithm to the representation of the electric field in $\omega$ space. We employ a masking function in the time domain to smoothly turn the pulses on and off. The ensemble average is a square pulse with rise and fall times equal to $1 \%$ of the 
total pulse duration $T$. The sampling width in $\omega$ space is $2 \pi / T$. The number of independent modes determines the temporal resolution. We choose $T=230$ fs [29] and include 12288 independent modes. In the second scenario, we simulate nonfluctuating, coherent pulses by employing for $\mathcal{E}_{\mathrm{c}}(t)$ a simple Gaussian temporal envelope with root-mean-square width $\sigma$. In this case, we set $\mathcal{E}_{\mathrm{s}}(t)=0$.

Let $\hat{H}_{0}$ denote the field-free atomic Hamiltonian, and let $\hat{z}$ stand for the $z$ component of the electric dipole operator. Then the Hamiltonian in the presence of the x-ray field is given by

$$
\hat{H}(t)=\hat{H}_{0}+\hat{z} \mathcal{E}(t) .
$$

Because of the compactness of the $1 s$ orbital in Ne in comparison to the $1 s \rightarrow 3 p$ resonance wavelength, nondipole effects are of minor importance and may be neglected. Using the electronic model space specified above, the state vector satisfying the time-dependent Schrödinger equation

$$
i \frac{\partial}{\partial t}|\Psi, t\rangle=\hat{H}(t)|\Psi, t\rangle
$$

may be written as

$$
|\Psi, t\rangle=\tilde{c}_{1}(t)|1\rangle+\tilde{c}_{2}(t)|2\rangle+\sum_{i} \int d \varepsilon_{i} \tilde{g}_{i}\left(\varepsilon_{i}, t\right)\left|i, \varepsilon_{i}\right\rangle .
$$

For the expansion coefficients appearing in Eq. (7) we make the ansatz

$$
\begin{gathered}
\tilde{c}_{1}(t)=c_{1}(t) e^{-i\left(E_{1}+\delta / 2\right) t}, \\
\tilde{c}_{2}(t)=c_{2}(t) e^{-i\left(E_{2}-\delta / 2\right) t}
\end{gathered}
$$

and

$$
\tilde{g}_{i}\left(\varepsilon_{i}, t\right)=g_{i}\left(\varepsilon_{i}, t\right) e^{-i\left(E_{2}-\delta / 2\right) t} e^{i \varphi_{i}} .
$$

Here, $E_{1}$ is the Ne ground-state energy, $E_{2}$ is the energy position of the resonance state (including any shifts due to Coulomb coupling between $|2\rangle$ and the $\left|i, \varepsilon_{i}\right\rangle$ ), and $\delta=E_{2}-$ $E_{1}-\omega_{\mathrm{x}}$ is the x-ray detuning with respect to the resonance transition energy.

We assume that the x-ray field couples only $|1\rangle$ and $|2\rangle$. Describing $|1\rangle$ in terms of a closed-shell Slater determinant and $|2\rangle$ in terms of a linear combination of two particlehole Slater determinants forming a spin-singlet state, the transition dipole matrix element between $|1\rangle$ and $|2\rangle$ is given by [42]

$$
\langle 2|\hat{z}| 1\rangle=\sqrt{2}\left\langle 3 p_{z}|\hat{z}| 1 s\right\rangle \approx 0.0096,
$$


where the $1 s$ and $3 p_{z}$ orbitals of Ne were calculated using the Hartree-Fock-Slater mean-field model [43]. (This strategy gives accurate absolute x-ray absorption cross sections near the Ne $K$ edge [37].) The direct x-ray-induced transition from $|1\rangle$ to any of the $\left|i, \varepsilon_{i}\right\rangle$ is neglected. Since $|2\rangle$ and $\left|i, \varepsilon_{i}\right\rangle$ have the same parity, $\left\langle 2|\hat{z}| i, \varepsilon_{i}\right\rangle=0$.

In order to obtain equations of motion for $c_{1}(t), c_{2}(t)$, and $g_{i}\left(\varepsilon_{i}, t\right)$, Eqs. $(7),(8),(9)$, and (10) may be inserted into Eq. (6). Upon making the rotating wave approximation [44] and introducing the complex Rabi frequency

$$
\mathcal{R}(t)=\langle 2|\hat{z}| 1\rangle\left\{\mathcal{E}_{\mathrm{c}}(t)+i \mathcal{E}_{\mathrm{s}}(t)\right\}
$$

it follows that the equation of motion for the ground-state amplitude reads

$$
i \dot{c}_{1}(t)=-\frac{\delta}{2} c_{1}(t)+\frac{\mathcal{R}^{*}(t)}{2} c_{2}(t) .
$$

Treating the decay of $|2\rangle$ into the manifold of the $\left|i, \varepsilon_{i}\right\rangle$ in the standard way [44], the equation of motion for the resonance-state amplitude is

$$
i \dot{c}_{2}(t)=-i \frac{\Gamma_{1 s^{-1}}}{2} c_{2}(t)+\frac{\delta}{2} c_{2}(t)+\frac{\mathcal{R}(t)}{2} c_{1}(t)
$$

with the total decay width

$$
\Gamma_{1 s^{-1}}=\sum_{i} \Gamma_{i}
$$

Finally, we use Eq. (2) to express the equation of motion for the amplitude for the decay channel $\left|i, \varepsilon_{i}\right\rangle$ in terms of the partial decay width $\Gamma_{i}$ :

$$
i \dot{g}_{i}\left(\varepsilon_{i}, t\right)=\left[E_{i}^{(+)}+\varepsilon_{i}-E_{2}+\frac{\delta}{2}\right] g_{i}\left(\varepsilon_{i}, t\right)+\sqrt{\frac{\Gamma_{i}}{2 \pi}} c_{2}(t) .
$$

We have introduced $E_{i}^{(+)}$to denote the channel threshold of the $i$ th channel (i.e., the energy of the $i$ th energetically accessible state of $\mathrm{Ne}^{+}$). As mentioned in Sec. II, we consider only electronically stable states of $\mathrm{Ne}^{+}$and therefore do not assign a decay width to them.

From Eq. (16) we may conclude that the resonant Auger electron line profile associated with the $i$ th channel is given by

$$
\begin{aligned}
P_{i}\left(\varepsilon_{i}\right) & =\lim _{t \rightarrow \infty}\left|g_{i}\left(\varepsilon_{i}, t\right)\right|^{2} \\
& =\frac{\Gamma_{i}}{2 \pi} \lim _{t \rightarrow \infty}\left|\int_{-\infty}^{t} d t^{\prime} c_{2}\left(t^{\prime}\right) e^{i\left[E_{i}^{(+)}+\varepsilon_{i}-E_{2}+\delta / 2\right] t^{\prime}}\right|^{2} .
\end{aligned}
$$


Note that while the area under the resonant Auger electron line profile is proportional to the partial width $\Gamma_{i}$, the shape of the resonant Auger electron line profile does not depend on $\Gamma_{i}$, but is dependent on the details of the time evolution of the amplitude of state $|2\rangle$ [cf. Eq. (14)]. Since for resonant Auger decay $E_{2} \gg E_{i}^{(+)}$in comparison to the decay width, the resonant Auger electron line shape is also independent of the channel threshold $E_{i}^{(+)}$. Therefore, within this model, the line profiles calculated in Sec. IV have the same shape for each of the resonant Auger channels discussed in Sec. II.

Throughout the rest of this paper we assume that $\omega_{\mathrm{x}}$ equals the energy separation between the atomic ground state and the resonance state, i.e., we set $\delta=0$. The equations of motion (13), (14), and (16) are solved utilizing a fourth-order Runge-Kutta integrator. Before the interaction with an x-ray pulse, the atom is assumed to be in its neutral ground state. Our numerical calculations are based on a transverse spatial pulse profile that is circular with a uniform intensity distribution inside the circle. Spatial averaging, which would be necessary for non-uniform intensity distributions, is not performed.

\section{NUMERICAL RESULTS}

At low x-ray intensity, the probability of depopulating the Ne ground state is small, and the transition from level $|1\rangle$ to level $|2\rangle$ may be treated perturbatively. Even as the intensity is increased, x-ray-induced emission from $|2\rangle$ back to $|1\rangle$ remains irrelevant as long as the resonant Auger decay of $|2\rangle$ is faster than the induced emission process. In order to induce Rabi oscillations [44] between levels $|1\rangle$ and $|2\rangle$ on a time scale that is comparable to the resonant Auger lifetime of level $|2\rangle$, the x-ray intensity required is

$$
I_{\min }=\frac{1}{8 \pi \alpha}\left(\frac{2 \pi \Gamma_{1 s^{-1}}}{\langle 2|\hat{z}| 1\rangle}\right)^{2} \approx 1.5 \times 10^{18} \frac{\mathrm{W}}{\mathrm{cm}^{2}} .
$$

This approximate minimum intensity needed for Rabi oscillations is so unusually high because Auger decay is ultrafast, taking place on a time scale of femtoseconds, and because transition dipole matrix elements involving an inner-shell electron are relatively small.

Assuming LCLS pulse parameters, it is possible to reach and even exceed $I_{\min }$. In Fig. 1a, we plot the time evolution of the Ne ground-state population during a typical chaotic $\mathrm{x}$ ray pulse [first scenario described in the paragraph following Eq. (4)]. The ground-state population drops rapidly during the first few radiation spikes, as a consequence of the x-ray- 


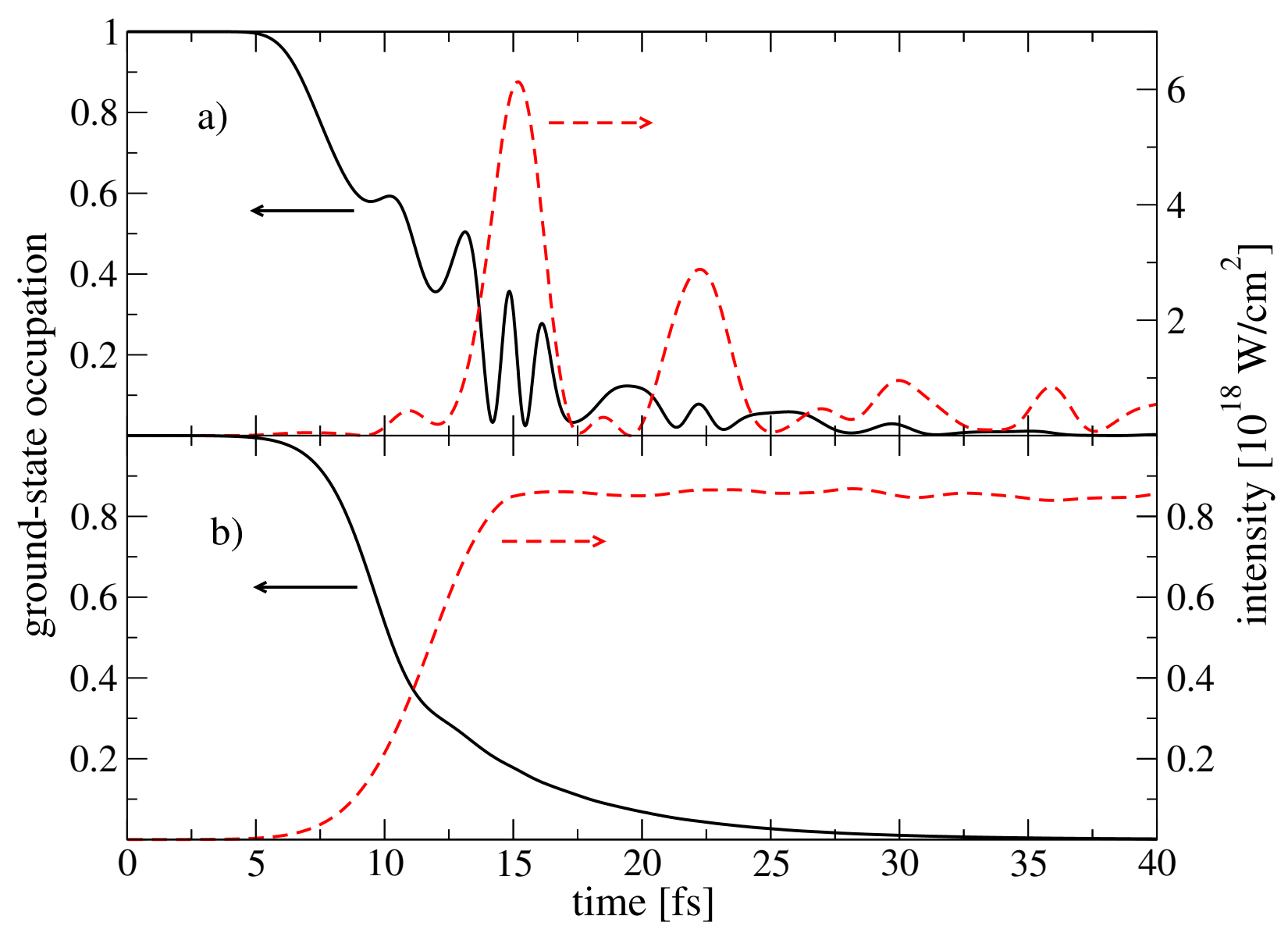

FIG. 1: (Color online) Time evolution of the x-ray intensity (red dashed line) and of the neon ground-state population (solid black line) for a single shot (a) and for the averaged ensemble (b). Parameters characterizing the x-ray pulse ensemble: pulse length 230 fs (FWHM); $10^{13}$ photons per pulse; focal diameter $1 \mu \mathrm{m}$.

induced excitation from the ground state to level $|2\rangle$ followed by the resonant Auger decay of level $|2\rangle$. Superimposed on the irreversible depletion of the ground-state population are Rabi oscillations with a period of the order of one femtosecond. Because of x-ray phase fluctuations, the chaotic x-ray pulse is not exactly in resonance with the $1 s \rightarrow 3 p$ transition. This is the reason why generally there is no complete population transfer from level $|1\rangle$ to level $|2\rangle$ during the Rabi oscillations.

In order to detect the x-ray-induced Rabi oscillations in Ne as suggested by Fig. 1a, one would have to measure - for an individual SASE free-electron laser pulse - the time evolution of the ground-state population (or, alternatively, of the resonant Auger electron yield) with 
sub-femtosecond time resolution. As shown in Fig. 1b, after averaging over an ensemble of 10000 chaotic x-ray pulses, the oscillatory behavior of the Ne ground-state population disappears. A time-domain measurement of the x-ray-induced population dynamics is therefore quite challenging.

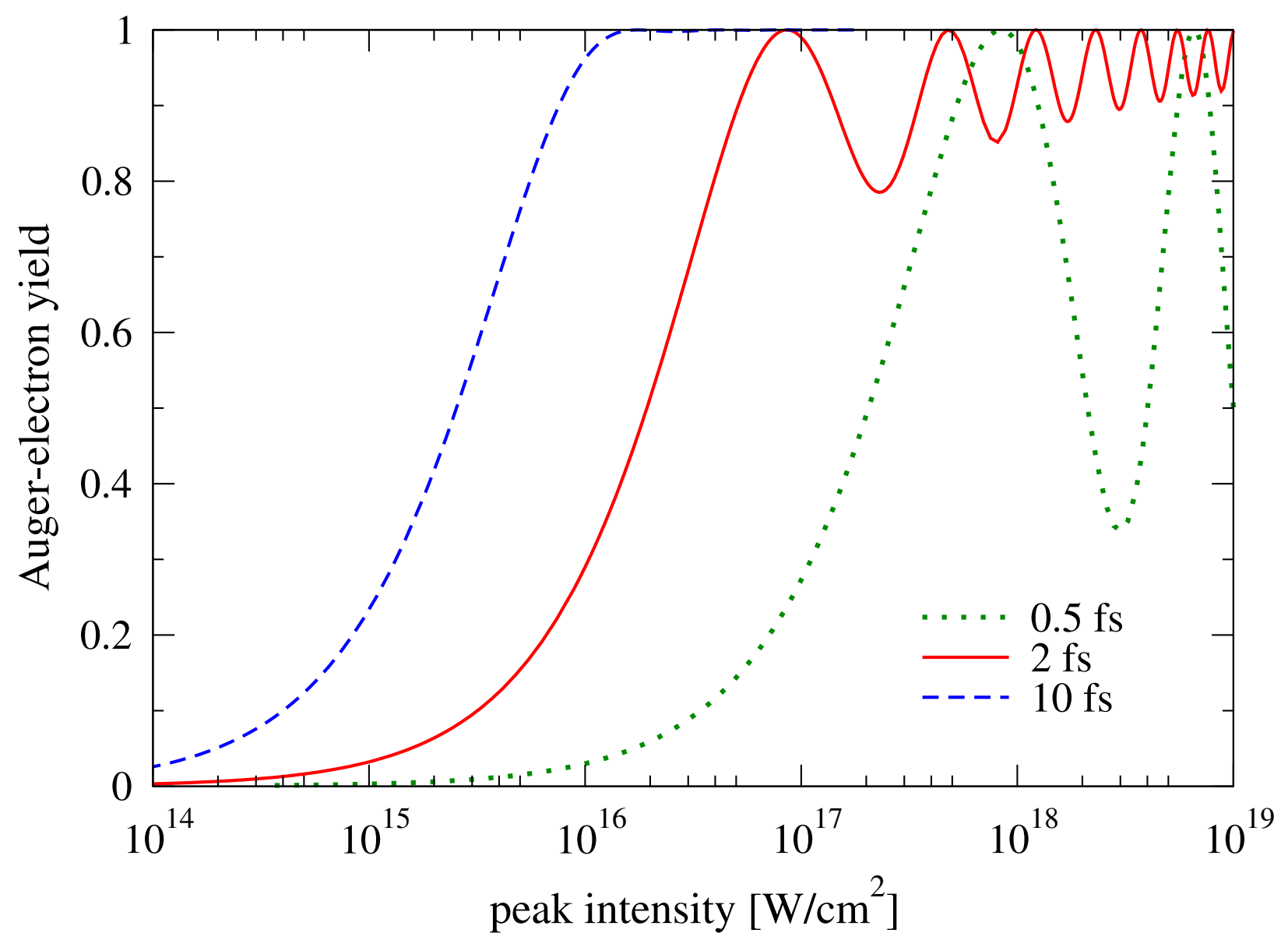

FIG. 2: (Color online) Total resonant Auger electron yield after exposure to a Gaussian-shaped pulse of duration $\sigma=0.5 \mathrm{fs}$ (green dotted line), $\sigma=2 \mathrm{fs}$ (red solid line), and $\sigma=10 \mathrm{fs}$ (blue dashed line) as a function of the x-ray peak intensity.

If the x-ray pulses are coherent and sufficiently short, a time-resolved measurement is, in fact, not necessary. This is illustrated in Fig. 2, where the total resonant Auger electron yield after exposure to a coherent $\mathrm{x}$-ray pulse [second scenario described in the paragraph following Eq. (4)] is plotted as a function of the x-ray peak intensity. The idea here is that for fixed pulse duration, the state $|\Psi, t\rangle$ reached at the end of the x-ray pulse will depend on the x-ray peak intensity. If the x-ray pulse is long in comparison with the resonant Auger 
lifetime ( $\sigma=10$ fs in Fig. 2), then even if Rabi oscillations take place during the x-ray pulse, by the time the pulse is over, the atom is ionized with a probability of one (cf. Fig. 1). However, when the pulses are sufficiently short ( $\sigma=0.5$ fs and $\sigma=2$ fs in Fig. 2), then, after the laser pulse is over, the atom has a finite probability of being in its ground state after having undergone one or more Rabi oscillations. As the intensity is increased, the first minimum in the resonant Auger electron yield appears when the atom manages to complete one Rabi cycle during the x-ray pulse ( $2 \pi$ pulse); the second minimum appears when the atom manages to complete two Rabi cycles during the x-ray pulse ( $4 \pi$ pulse), and so forth.

As shown in Fig. 3 for $\sigma=2$ fs, Rabi flopping induced by a coherent x-ray pulse also leads to a marked modification of the resonant Auger electron line profile associated with a given resonant Auger channel. At an x-ray peak intensity of $8.6 \times 10^{16} \mathrm{~W} / \mathrm{cm}^{2}$, corresponding to a $\pi$ pulse, the resonant Auger electron line profile consists of a single peak (Fig. 3a). A fit reveals that this peak is more similar to a Gaussian than to a Lorentzian. Even when fitted to a Lorentzian, the width (FWHM) obtained is only $0.20 \mathrm{eV}$, which is smaller than $\Gamma_{1 s^{-1}}$. This is consistent with the fact that for the pulse duration chosen here, the spectral bandwidth of the coherent x-ray pulse is smaller than the Auger width; for a $\pi$ pulse, the width of the resonant Auger electron line profile is largely determined by the $\mathrm{x}$-ray bandwidth. However, if $n \pi$ pulses with $n>1$ are chosen, the resonant Auger electron line profile does not resemble either a Gaussian or a Lorentzian and is substantially broader than either the x-ray bandwidth or the Auger width. This is illustrated in Fig. 3a for $3 \pi\left(4.9 \times 10^{17} \mathrm{~W} / \mathrm{cm}^{2}\right)$ and $5 \pi\left(1.3 \times 10^{18} \mathrm{~W} / \mathrm{cm}^{2}\right)$ pulses, and in Fig. $3 \mathrm{~b}$ for $2 \pi(2.4 \times$ $\left.10^{17} \mathrm{~W} / \mathrm{cm}^{2}\right), 4 \pi\left(8.3 \times 10^{17} \mathrm{~W} / \mathrm{cm}^{2}\right)$, and $6 \pi\left(1.7 \times 10^{18} \mathrm{~W} / \mathrm{cm}^{2}\right)$ pulses. For both $(2 n+1) \pi$ $[n=0,1, \ldots]$ and $2 n \pi[n=1,2, \ldots]$ pulses, the resonant Auger electron line profile displays $n+1$ local maxima. Such a multipeak electron spectrum for a short, intense laser pulse was predicted more than 20 years ago [46]. An analogous multipeak effect should exist in resonance fluorescence [47] and has been interpreted as a temporal diffraction phenomenon $[48]$.

We would like to point out that the width (FWHM) of the multipeak line profiles plotted in Fig. 3 approximately tracks the peak Rabi frequency during the respective $\mathrm{x}$-ray pulse and thus is a measure of the x-ray peak electric field amplitude. For instance, at a peak intensity of $4.9 \times 10^{17} \mathrm{~W} / \mathrm{cm}^{2}$, the peak Rabi frequency is $1.0 \mathrm{eV}$, which agrees approximately with the width of the corresponding line profile shown in Fig. 3a. The peak Rabi frequency at a 


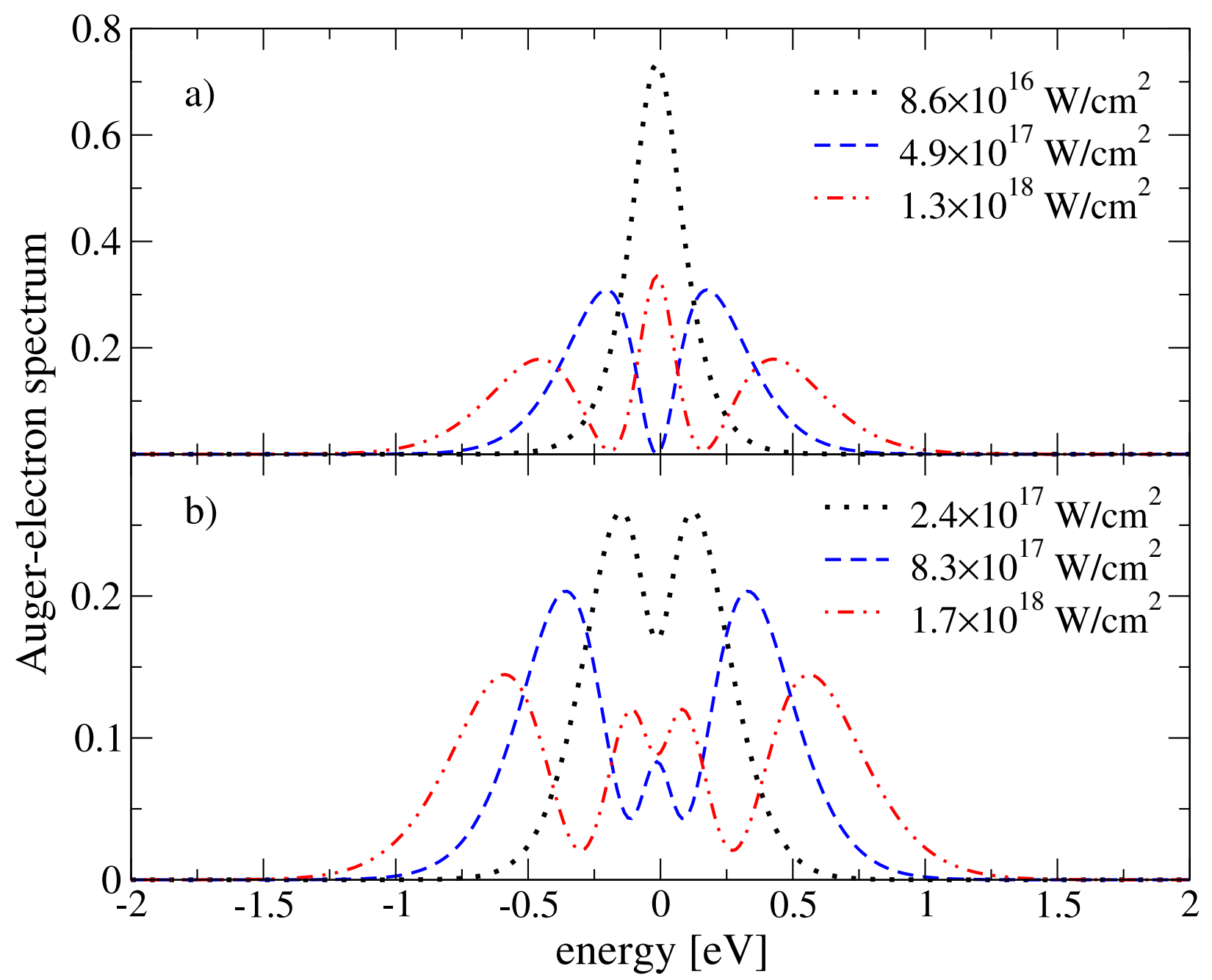

FIG. 3: (Color online) Resonant Auger electron line profile for a Gaussian-shaped pulse of duration $\sigma=2$ fs for different peak intensities. The peak intensities chosen correspond, respectively, to the first three maxima (a) and the first three minima (b) of the total resonant Auger electron yield depicted in Fig. 2.

peak intensity of $1.7 \times 10^{18} \mathrm{~W} / \mathrm{cm}^{2}$ is $1.8 \mathrm{eV}$, in approximate agreement with Fig. $3 \mathrm{~b}$.

The multipeak structure in resonant electron or fluorescence spectra has apparently not yet been experimentally observed [49]. Part of the problem may be that precise control of the pulse area is required [49]. (Another practical difficulty is volume averaging over the spatially non-uniform intensity profile of the laser beam.) Using a SASE free-electron laser such as LCLS, control of the pulse area will not be available. We therefore compare in Fig. 4 resonant Auger electron line profiles obtained for a single chaotic x-ray pulse (Fig. 4a) and 


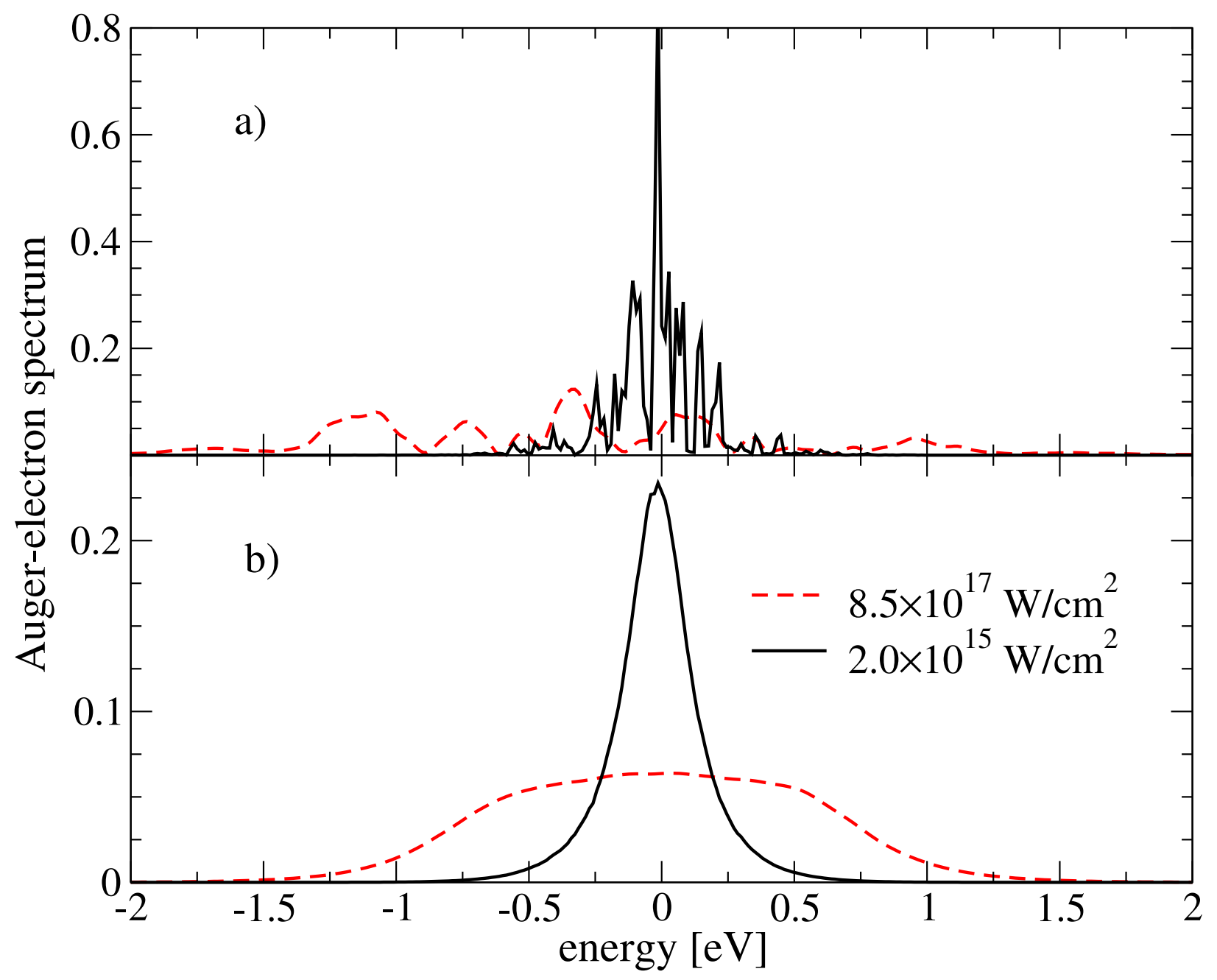

FIG. 4: (Color online) Resonant Auger electron line profiles for a single shot (a) and after averaging over an ensemble of 10000 shots (b). The red dashed lines are results for an average peak intensity of $8.5 \times 10^{17} \mathrm{~W} / \mathrm{cm}^{2}$ (focal diameter of $1 \mu \mathrm{m}$ with temporal intensity distribution for single shot corresponding to Fig. 1). Solid black lines correspond to an average peak intensity of $2.0 \times 10^{15} \mathrm{~W} / \mathrm{cm}^{2}$ (focal diameter of $21 \mu \mathrm{m}$ ). A pulse duration of $230 \mathrm{fs}$ and $10^{13}$ photons per pulse are assumed.

for an ensemble of 10000 chaotic x-ray pulses (Fig. 4b). The single-shot electron line profile at low intensity $\left(2.0 \times 10^{15} \mathrm{~W} / \mathrm{cm}^{2}\right.$ in Fig. 4$)$ is extremely spiky and reflects the multimode nature of a chaotic radiation pulse. When averaged over many shots, the resonant Auger electron line profile can be approximated by a Lorentzian with a width (FWHM) of $0.24 \mathrm{eV}$, which is $\sim 10 \%$ less than $\Gamma_{1 s^{-1}}$. However, if the x-ray intensity is high enough to induce 
Rabi oscillations within a resonant Auger lifetime $\left(8.5 \times 10^{17} \mathrm{~W} / \mathrm{cm}^{2}\right.$ in Fig. 4), both the single-shot electron line profile and the ensemble average are substantially broader than $\Gamma_{1 s^{-1}}$. Thus, even without control over the pulse area, the measurement of resonant Auger electron line profiles could be used to indirectly detect the presence of x-ray-driven Rabi oscillations. It would be particularly interesting if single-shot measurements of resonant Auger electron line profiles were feasible. As can be seen in Fig. 4a, at high x-ray intensity the single-shot electron line profile is much smoother than at low intensity, and a nodal structure reminiscent of the multipeak structures in Fig. 3 is discernible.

\section{CONCLUSION}

In this paper, we have employed an effective two-level model to investigate the impact of resonant high-intensity x-ray radiation on the resonant Auger effect of atomic neon at the $1 s \rightarrow 3 p$ transition. This model assumes, for instance, that $\mathrm{x}$-ray absorption by valence electrons may be neglected in comparison to the strong coupling between levels $|1\rangle$ and $|2\rangle$. We have also neglected AC Stark shifts due to electronic states outside the model space. Estimates indicate that these effects are relatively small and should not affect the general conclusions of this paper. A comprehensive analysis of these effects will be a subject of future investigations.

As shown in the previous section, detecting x-ray-induced Rabi oscillations using a SASE free-electron laser by measuring the time evolution of the Ne ground-state population (or of the resonant Auger electron yield) is challenging. Besides requiring single-shot measurements, this would necessitate sub-femtosecond temporal resolution. In principle, it is possible to measure the peak-intensity dependence of the resonant Auger electron yield. However, the resonant Auger electron yield as a function of the peak intensity will display oscillations associated with Rabi flopping in the time domain only if the x-ray pulses are ultrashort (shorter than the Auger lifetime) and fully longitudinally coherent. As an alternative, we recommend to perform spectroscopy of the resonant Auger electron line profile. For ultrashort, coherent $\mathrm{x}$-ray pulses, one would expect to see interesting multipeak effects as soon as the x-ray intensity is high enough to induce Rabi oscillations. Hints of such multipeak effects are still visible for individual chaotic pulses. After averaging over many chaotic x-ray pulses, the resonant Auger electron line profile at high x-ray intensity is structureless, but is 
substantially broadened relative to the resonant Auger electron line profile obtained at low x-ray intensity. The width of the resonant Auger electron line profile allows one to estimate the peak electric field amplitude, and thus the peak intensity, for x rays of sufficiently high intensity. It may therefore be possible to exploit the resonant Auger effect as an effective diagnostic tool for x-ray free-electron lasers.

\section{Acknowledgments}

We thank P. Bucksbaum, C. Buth, E. Kanter, B. Krässig, S. Southworth, and L. Young for inspiring discussions. This work was supported by the Office of Basic Energy Sciences, Office of Science, U.S. Department of Energy, under Contract No. DE-AC02-06CH11357. Part of this work was performed under the auspices of the U.S. Department of Energy by Lawrence Livermore National Laboratory under Contract DE-AC52-07NA27344.

[1] G. S. Brown, M. H. Chen, B. Crasemann, and G. E. Ice, Phys. Rev. Lett. 45, 1937 (1980).

[2] G. B. Armen, H. Aksela, T. Åberg, and S. Aksela, J. Phys. B 33, R49 (2000).

[3] F. Gel'mukhanov and H. Ågren, Phys. Rep. 312, 87 (1999).

[4] E. M. Garcia, A. Kivimäki, L. G. M. Pettersson, J. A. Ruiz, M. Coreno, M. de Simone, R. Richter, and K. C. Prince, Phys. Rev. Lett. 96, 063003 (2006).

[5] M. Grimm, B. Langer, S. Schlemmer, T. Lischke, U. Becker, W. Widdra, D. Gerlich, R. Flesch, and E. Rühl, Phys. Rev. Lett. 96, 066801 (2006).

[6] O. Kugeler, G. Prumper, R. Hentges, J. Viefhaus, D. Rolles, U. Becker, S. Marburger, and U. Hergenhahn, Phys. Rev. Lett. 93, 033002 (2004).

[7] P. O’Keeffe, S. Aloise, M. Meyer, and A. N. Grum-Grzhimailo, Phys. Rev. Lett. 90, 023002 (2003).

[8] J. Danger, P. Le Fevre, H. Magnan, D. Chandesris, S. Bourgeois, J. Jupille, T. Eickhoff, and W. Drube, Phys. Rev. Lett. 88, 243001 (2002).

[9] M. Meyer, S. Aloise, and A. N. Grum-Grzhimailo, Phys. Rev. Lett. 88, 223001 (2002).

[10] R. Feifel, F. Burmeister, P. Salek, M. N. Piancastelli, M. Bassler, S. L. Sorensen, C. Miron, H. Wang, I. Hjelte, O. Bjorneholm, A. N. de Brito, F. K. Gel'mukhanov, H. Ågren, and S. 
Svensson, Phys. Rev. Lett. 85, 3133 (2000).

[11] K. Ueda, Y. Shimizu, H. Chiba, Y. Sato, M. Kitajima, H. Tanaka, and N. M. Kabachnik, Phys. Rev. Lett. 83, 5463 (1999).

[12] K. Ueda, M. Simon, C. Miron, N. Leclercq, R. Guillemin, P. Morin, and S. Tanaka, Phys. Rev. Lett. 83, 3800 (1999).

[13] E. Pahl, L. S. Cederbaum, H.-D. Meyer, and F. Tarantelli, Phys. Rev. Lett. 80, 1865 (1998).

[14] H. Aksela, M. Kivilompolo, E. Nõmmiste, and S. Aksela, Phys. Rev. Lett. 79, 4970 (1997).

[15] E. Kukk, H. Aksela, S. Aksela, F. Gel'mukhanov, H. Ågren, and S. Svensson, Phys. Rev. Lett. 76, 3100 (1996).

[16] S. Aksela, E. Kukk, H. Aksela, and S. Svensson, Phys. Rev. Lett. 74, 2917 (1995).

[17] A. Kivimäki, A. Naves de Brito, S. Aksela, H. Aksela, O. P. Sairanen, A. Ausmees, S. J. Osborne, L. B. Dantas, and S. Svensson, Phys. Rev. Lett. 71, 4307 (1993).

[18] C. Pellegrini and S. Reiche, IEEE J. Select. Top. Quant. Electr. 10, 1393 (2004).

[19] J. Feldhaus, J. Arthur, and J. B. Hastings, J. Phys. B 38, S799 (2005).

[20] J. Arthur et al., Linac Coherent Light Source (LCLS) Conceptual Design Report, SLAC-R-593 (2002). http://www-ssrl.slac.stanford.edu/lcls/cdr/

[21] T. Tanaka and T. Shintake (Eds.), SCSS X-FEL Conceptual Design Report (Riken, Japan, May 2005). http://www-xfel.spring8.or.jp/

[22] M. Altarelli et al. (Eds.), The European X-ray Free-Electron Laser, Technical Design Report, DESY 2006-XXX (2006). http://xfel.desy.de/tdr/index_eng.html

[23] LCLS: The first experiments, http://www-ssrl.slac.stanford.edu/lcls/papers/lcls_ experiments_2.pdf

[24] R. Bonifacio, C. Pellegrini, and L. M. Narducci, Opt. Commun. 50, 373 (1984).

[25] Y. Li, S. Krinsky, J. W. Lewellen, K.-J. Kim, V. Sajaev, and S. V. Milton, Phys. Rev. Lett. 91, 243602 (2003).

[26] W. Ackermann et al., Nature Photonics 1, 336 (2007).

[27] S. Krinsky and R. L. Gluckstern, Phys. Rev. ST AB 6, 050701 (2003).

[28] N. Rohringer and R. Santra, Phys. Rev. A 76, 033416 (2007).

[29] LCLS parameters may be found at http://www-ssrl.slac.stanford.edu/htbin/rdbweb/ LCLS_params_DB_public/.

[30] V. Schmidt, Electron spectrometry of atoms using synchrotron radiation (Cambridge Univer- 
sity Press, Cambridge, 1997).

[31] A. Kivimäki, S. Heinäsmäki, M. Jurvansuu, S. Alitalo, E. Nõmmiste, H. Aksela, and S. Aksela, J. Electron Spectrosc. Relat. Phenom. 114-116, 49 (2001).

[32] Y. Shimizu, H. Yoshida, K. Okada, Y. Muramatsu, N. Saito, H. Ohashi, Y. Tamenori, S. Fritzsche, N. M. Kabachnik, H. Tanaka, and K. Ueda, J. Phys. B 33, L685 (2000).

[33] M. Coreno, L. Avaldi, R. Camilloni, K. C. Prince, M. de Simone, J. Karvonen, R. Colle, and S. Simonucci, Phys. Rev. A 59, 2494 (1999).

[34] T. Hayaishi, E. Murakami, Y. Morioka, E. Shigemasa, A. Yagishita, and F. Koike, J. Phys. B 28, 1411 (1995).

[35] H. Aksela, S. Aksela, J. Tulkki, T. Åberg, G. M. Bancroft, and K. H. Tan, Phys. Rev. A 39, 3401 (1989).

[36] T. W. Gorczyca, Phys. Rev. A 61, 024702 (2000).

[37] C. Buth, R. Santra, and L. Young, Phys. Rev. Lett. 98, 253001 (2007).

[38] V. F. Weisskopf and E. P. Wigner, Z. Phys. 63, 54 (1930).

[39] R. Santra and L. S. Cederbaum, Phys. Rep. 368, 1 (2002).

[40] G. Vannucci and M. C. Teich, Appl. Opt. 19, 548 (1980).

[41] The coherence time is given by $\tau=\sqrt{\pi} / \sigma_{\omega}$, where $\sigma_{\omega}$ is the SASE gain bandwidth [27]. Using Refs. [27, 29], we estimate that the gain bandwidth at $800 \mathrm{eV}$ is $0.35 \mathrm{eV}$, resulting in a coherence time of 3.3 fs.

[42] See, for example, N. Rohringer, A. Gordon, and R. Santra, Phys. Rev. A 74, 043420 (2006).

[43] F. Herman and S. Skillman, Atomic Structure Calculations (Prentice-Hall, Englewood Cliffs, N.J., 1963).

[44] P. Meystre and M. Sargent III, Elements of Quantum Optics (Springer, Berlin, 1991).

[45] C. P. Bhalla, N. O. Folland, and M. A. Hein, Phys. Rev. A 8, 649 (1973).

[46] K. Rzazewski, J. Zakrzewski, M. Lewenstein, and J. W. Haus, Phys. Rev. A 31, 2995 (1985).

[47] M. Lewenstein, J. Zakrzewski, and K. Rzazewski, J. Opt. Soc. Am. B 3, 22 (1986).

[48] E. J. Robinson, J. Phys. B 19, L657 (1986).

[49] M. Lewenstein, private communication. 